\title{
Special Issue on Spin Statistics
}

\author{
Edoardo Milotti • C. Curceanu (Petrascu) • \\ Stephen L. Adler • Michael Berry
}

Published online: 5 February 2010

(C) Springer Science+Business Media, LLC 2010

\section{1 “Introductory Comments" by Edoardo Milotti and Catalina Curceanu}

A workshop dedicated to spin statistics - "SpinStat2008"-was held at the end of October 2008 at the Stazione Marittima Conference Center in Trieste, Italy: it was meant to focus especially on experimental and theoretical aspects of the spin-statistics connection and of related symmetries (in particular the CPT and the Lorentz symmetries). The workshop was quite successful and everybody there felt that there should be a follow-up, and that the many interesting contributions and ideas presented there should be put in paper form. After some thinking we decided that the best format would be a series of refereed papers in a topical issue of an outstanding journal, open to all contributors, rather than the usual volume of conference papers restricted to participants. The Editors of Foundations of Physics kindly accepted to host this topical issue, and the ensuing call for papers drew a respectable flow of interesting and novel ideas, and some very appealing review papers. The comments that follow have been

E. Milotti (凶)

Dipartimento di Fisica and INFN - Sezione di Trieste, Via Valerio 2, 34127 Trieste, Italy

e-mail: edoardo.milotti@ts.infn.it

C. Curceanu (Petrascu)

INFN, Laboratori Nazionali di Frascati, CP 13, Via E. Fermi 40, 00044 Frascati (Roma), Italy

e-mail: petrascu@lnf.infn.it

S.L. Adler

Institute for Advanced Study, School of Natural Sciences, Einstein Drive, Princeton, NJ 08540, USA

e-mail: adler@ias.edu

M. Berry

H.H. Wills Physics Laboratory, University of Bristol, Tyndall Avenue, Bristol BS8 1TL, UK

e-mail: tracie.anderson@bristol.ac.uk 
contributed by two eminent physicists who took part to the workshop and agreed to have their say on the topic and on the liveliness of the field. Now we look forward to further exciting meetings and both experimental and theoretical ideas, because we still feel that some deep physics lies concealed by the shroud of Pauli's Principle; Pauli himself strongly expressed this idea in his Nobel lecture: "already in my original paper I stressed the circumstance that I was unable to give a logical reason for the exclusion principle or to deduce it from more general assumption. I had the feeling and I still have it today, that this is a deficiency. The impression that the shadow of some incompleteness fell here on the bright light of success of the new quantum mechanics seems to me unavoidable".

\section{2 “An Appreciation of the Workshop SpinStat 2008” by Stephen Adler}

The spin-statistics connection grew historically out of the Pauli exclusion principle and its application in understanding atomic structure, and later, nuclear structure. Its modern ramifications take many forms, which were admirably reviewed at the recent workshop on "Theoretical and experimental aspects of the spin-statistics connection and related symmetries".

First of all, experimental tests of the Pauli principle were extensively covered. These take the form of searches for possible very small deviations from the selection rules that are required by the exclusion principle, in both nuclear and atomic systems. So far no deviations have been found, and very stringent limits on possible Pauli principle violations have been set. A theoretical counterpart of the experimental search for Pauli principle violations is the formulation of theoretical frameworks, going beyond standard quantum mechanics and quantum field theory, in which such violations are permitted. One goal here is to have alternative theories, not obeying the Pauli principle, to use as "foils" against which to test the standard predictions, by giving theoretically well-defined parameters governing Pauli principle violation, which can be bounded in various experimental tests. Another is to achieve a deeper understanding of the spin-statistics connection by examining its analogs, and lack thereof, in non-conventional systems.

Aspects of this discussed at the workshop included q-modified commutation relations, stringy theories, and non-commutative geometries. The mathematical foundations of the Pauli principle, and more generally, of the spin-statistics connection, continue to be an actively pursued subject. Questions under current investigation include simplified derivations of the spin-statistics connection, such as ones which use input only from non-relativistic quantum mechanics, and analogs of the spin-statistics connection in higher dimensions.

Other mathematical aspects represented at the workshop included group theoretical underpinnings of the angular momentum algebra and of Bose and Fermi statistics, discrete symmetries (such as CPT), statistics in the Moyal plane, and entanglement in quantum systems. Other topics peripherally related to the spin-statistics connection were also discussed. In particular, recent searches for dark matter, which is nearly five times as abundant in the universe as is ordinary baryonic matter, have yet to establish whether dark matter is bosonic, as in the supersymmetric models for cold dark matter, 
or is fermionic. The status of the dark matter search in the DAMA/LIBRA experiment was summarized, as were implications of possible dark matter bound to objects in the solar system. Finally, the Pauli principle, and more generally the spin-statistics connection, is now a subject of active study within the history and philosophy of science community, and this aspect was also represented at the workshop. Overall, the workshop gave a good view of the current status of a number of active and significantly overlapping areas of current research in mathematical, theoretical, and experimental physics, all related to the common theme of the bipartite division of the observed structure of matter into families of bosons and fermions.

\section{3 "Some Reflections After the Meeting" by Michael Berry}

The Stazione Marittima Conference Center, beautifully located on the Adriatic sea in downtown Trieste, was the perfect venue for an in-depth study of the spin-statistics connection (S-S). This aspect of our current physics is fundamental yet rarely considered. A surprise to me was the intellectual range of the papers and activities at the meeting; I had not appreciated that S-S had so many and such varied ramifications.

On the theoretical side, the main topics were:

- interpolating between the Bose (symmetric) and Fermi (antisymmetric) cases with a parameter $q$ that is $+1 /-1$ (Bose, Fermi) but which can take intermediate values, with the aim of interpreting experiments to test $\mathrm{S}-\mathrm{S}$;

- new results on intricate group-theoretical aspects of S-S;

- the role of single-valuedness in nonrelativistic attempts to obtain S-S by incorporating quantum exchange into the configuration space;

- S-S in quantum field theory, superstring theory and gravity;

- relation between the unavoidable entanglement implied by S-S and entanglement as a quantum information resource;

- proposal to test the possibility that S-S might not be established instantaneously.

I expected that many of these theoretical topics would be discussed at the meeting. What I did not anticipate was that many experiments, actual and proposed, to test possible violations of S-S, would be reviewed and described in detail. These involved optical spectroscopy, electron capture by ions, radioisotopes, neutral kaons, nucleons and neutrinos. Unsurprisingly, no violations have been detected so far.

The identicalness (indistinguishability) underlying $\mathrm{S}-\mathrm{S}$ in quantum physics breathes new life into ancient philosophical conundrums, at least as old as Leibniz's 'identity of indiscernibles'. A paper exploring these aspects was very well received.

One evening was devoted to explaining the subject of the meeting to local people who are not scientists. This began with a lecture giving the historical background to $\mathrm{S}-\mathrm{S}$. There followed readings in Italian from Russell Stannard's 'The new world of Mr Tompkins' (an updating of George Gamow's classic 'Mr Tompkins in Wonderland'), with a commentary (in English, translated into Italian) by a conference participant (me) playing the part of the Professor in the book - a surreal theatrical event, to which the audience responded with enthusiasm.

The chair and co-chairs Edoardo Milotti and Catalina Curceanu, and the other members of the Committee, are to be congratulated for such a thoughtfully planned, faultlessly organized and enlightening meeting. 